\title{
The effect of fracture roughness on the onset of non-linear flow
}

\section{Cunningham ${ }^{1}$, H. Auradou' ${ }^{2}$ S. Shojaei-Zadeh ${ }^{3}$ and G. Drazer ${ }^{1}$}

${ }^{1}$ Mechanical and Aerospace Engineering, Rutgers, The State University of New Jersey, Piscataway, NJ, USA.

${ }^{2}$ Université Paris-Saclay, CNRS, FAST, 91405, Orsay, France.

${ }^{3}$ National Science Foundation, 2415 Eisenhower Avenue, Alexandria, Virginia 22314, USA.

Corresponding author: German Drazer (german.drazer@rutgers.edu)

\section{Key Points:}

- Surface roughness leads to an early onset of inertial effects

- Surface roughness leads to an increase in spatial pressure fluctuations

- $\quad$ The critical Reynolds numbers correlates with normalized surface roughness

\begin{abstract}
In fractures where surface fluctuations are large compared to their aperture (narrow fractures) the flow is forced to move in tortuous paths that produce additional viscous friction and are subject to inertia effects. We consider the relation between the magnitude of surface roughness and the onset of inertial effects in the pressure driving the flow through a single open fracture. We performed experiments systematically varying the average aperture of the open fracture and covering a wide range of Reynolds numbers. For each aperture, we analyze the data in terms of the Forchheimer equation and show that the critical Reynolds number, defined as the Reynolds number at which inertial effects contribute $10 \%$ of the total pressure losses is highly correlated with the roughness of the surface. In particular, we show that significant inertial effects appear early as the relative importance of surface roughness increases. Finally, we present results showing that the magnitude of the deviations in the pressure field compared to a linear profile, taken at different points in the fracture along the flow direction, are directly related to the relative surface roughness of the fracture.
\end{abstract}




\section{Introduction}

Flow in fractured media is an important process in a broad range of engineering applications including hydrocarbon recovery, subsurface waste repositories and geothermal reservoir exploitation. When open fractures and fracture networks are present in low permeability rocks, they create long-range preferential pathways for fluids that would otherwise remain mostly immobile. As a result, for example, hydraulic fracturing is a common approach to enhance the flow of natural oil and gas. In general, the presence of fractures is key to a variety of phenomena that depend on the underground transport of fluids in rock formations, including $\mathrm{CO} 2$ sequestration and the dispersion of chemical and radioactive contaminants among others (National Research Council, 1996).

When fractures are narrow, that is, when surface fluctuations (or deviations from a flat surface) are large compared to the average aperture, both the magnitude of such surface fluctuations (surface roughness) and the contact area between the two surfaces distort the flow, forcing it to move in tortuous paths that produce additional viscous friction. In principle, the information on the characteristic aperture and surface roughness of the fractures could be inferred from outcrops or samples collected from the field. However, these methods require the reconstruction of the fracture void geometries - which generate uncertainties - and a flow model to relate the geometry of the fracture to its hydraulic properties. Alternatively, hydraulic tests can be performed in the field, but information is typically limited to macroscopic flow rates and global pressure drops along the fractures. Therefore, it would be a great advantage to be able to characterize fracture properties in addition to their hydraulic aperture, in particular, a measure of surface roughness and its relation to inertial flow effects, from simple flow rate versus pressure drop measurements. The purpose of our work is, in fact, to explore the importance of additional pressure losses due to inertial effect on the hydraulic properties of a single fracture.

At low Reynolds numbers, when inertia effects in the flow can be neglected, the relation between the flow rate through the fracture and the pressure gradient driving the flow is linear, as given by Darcy's law (Bear, 1988),

$$
q=\frac{-k_{f}}{\mu} \nabla p
$$

where $k_{f}$ is the hydraulic permeability of the fracture, $\mu$ is the fluid viscosity and $q$ is the specific discharge or superficial velocity of the flow. In the case of an open fracture, the superficial velocity is identical to the average velocity of the fluid $u=Q / A_{\mathrm{f}}$, where $Q$ is the flow rate and $A_{\mathrm{f}}$ is the cross-sectional area of the fracture perpendicular to the flow direction. Moreover, in the case of a completely smooth fracture, the flow corresponds to that between two parallel plates with a constant aperture $h$ (the separation between the two plates), and the hydraulic permeability can be calculated analytically from the Navier-Stokes equations, $k_{i v_{i}=h^{2} / 12 i}$. Therefore, if we approximate the flow in a fracture as that between parallel plates the dependence of the flow rate on the pressure gradient is then given by the so-called cubic-law (Witherspoon et al., 1980; Schrauf \& Evans, 1986; Brown, 1987),

$$
Q_{i \vee i=-L_{y} \frac{h^{3}}{12 \mu} \nabla p_{i \vee i i} i}
$$

where $L_{y}$ is the width of the fracture and $A_{f}=h \times L_{y}$.

In real fractures, however, the above expressions do not account for the effects of surface roughness on the flow field. When roughness is present and is not negligible compared to the aperture, it forces the fluid to follow tortuous paths (Brown, 1987). Clearly, the effect of surface roughness on the permeability depends on its relative magnitude compared to the aperture, which 
in underground fractures depends, in general, on the confining stress (Gangi, 1978). Numerous experimental and numerical studies have investigated the effect that roughness has on the hydraulic permeability of fractures (Drazer \& Koplik, 2000, 2002; H. Auradou et al., 2005, 2006; L. Talon et al., 2010; Laurent Talon et al., 2010; Mourzenko et al., 2018). In analogy with the cubic-law, a simple way to characterize the effect that surface roughness has on the permeability of the fracture is to define the hydraulic or effective aperture, $h_{\mathrm{eff}}$,

$$
Q=-L_{y} \frac{h_{e f f}^{3}}{12 \mu} \nabla p,
$$

where $h_{\text {eff }}$ is therefore the aperture between two parallel plates that would have the same permeability as the fracture under consideration. The effect of surface roughness can then be quantified by the ratio of the effective aperture to the average one, $h_{\mathrm{eff}} / h_{\mathrm{m}}$, where the average aperture is the mean separation between the surfaces of the fracture. A recent study also looked at spatial fluctuations of the local pressure and its connection with the tortuosity of the flow (Aminpour et al., 2018). Most of the studies characterizing roughness effects are intrinsically focused on low Reynolds number flows, also called Darcy flows or Stokes flows, in which inertia effects are negligible, and Darcy's law is valid.

On the other hand, the presence of tortuous paths induced by the roughness of the fracture may also result in an early onset of inertia effects in the flow field, and a nonlinear relation between the pressure gradient and the flow rate. More than a century ago, Forchheimer proposed an empirical quadratic relationship to describe the pressure gradient as a function of flow rate in porous media when inertia effects are present (Bear, 1988; Macdonald et al., 1979). The same relationship has been used in numerous studies to describe the flow in fractures at Reynolds numbers greater than one, and it is usually written as,

$$
\nabla p=A Q+B Q^{2}
$$

where $A$ and $B$ constants independent of the flow rate. Clearly, $A=12 \mu / L_{y} h_{\text {eff }}^{3}$ and the second term is the correction to Darcy's law to account for inertia effects in the flow. We note that we are assuming that Eq. (4) is valid for the entire range of Reynolds numbers (Skjetne et al., 1999; Zimmerman et al., 2004). We can re-write the equation in dimensionless form in terms of the Reynolds number of the flow $\mathfrak{R}=\rho Q / \mu L_{y}$, where $\rho$ is the density of the fluid, and the Forchheimer number Fo,

$$
\frac{\nabla p}{i \dot{i} \dot{b}}
$$

where the Forchheimer number, Fo, is defined as the ratio between the quadratic and linear terms, which can be interpreted as the ratio of non-linear, inertial contributions to the pressure drop, to the linear term accounting for viscous resistance (Macdonald et al., 1979). In any case, it is clear that the Forchheimer number gives the magnitude of the relative deviation from the linear regime and it is possible to use it to define the onset of non-linear flow (Javadi et al., 2014). In the previous equation, we have also introduced the dimensionless Forchheimer coefficient $\beta$ (Zimmerman et al., 2004), which, in principle, only depends on the geometry of the fracture and not on the flow conditions or the fluid properties.

As we mentioned, we can use the Forchheimer number to define a critical Reynolds number, $\mathfrak{R}_{c}$, as the value of the Reynolds number at which the pressure drop due to the nonlinear inertial contribution reaches a given fraction $\alpha$ of the total,

$$
\alpha=\frac{F O}{1+F o}=\frac{\beta \Re_{c}}{1+\beta \Re_{c}},
$$


where a $10 \%$ deviation is typically used, that is $\alpha=0.1 ; F o=1 / 9$ and $\Re_{c}=F o / \beta$. Therefore, the critical Reynolds number is expected to depend only on the geometry of the fracture and it could provide information on fracture roughness and flow tortuosity besides the reduction in permeability characterized by the ratio of effective to average apertures.

Only in recent years some work has focused on exploring the values of the critical Reynolds number in fractures, and in many cases, its correlation with surface roughness was not considered. In Table 1, we present a summary of representative results from the literature. Zimmerman et al. performed experiments with a rough fracture brought to contact and obtained a critical Reynolds number $\mathfrak{R}_{c} \sim 10$, but mentioned that this value would decrease with increasing fracture roughness (Al-Yaarubi, 2003; Zimmerman et al., 2004). Similar values of the critical Reynolds number $\left(\mathfrak{R}_{c} \sim 5-30\right)$ were observed by Rajith and Darlington (2007), Ji et al. (2008) and Radilla et al. (2013) in granite fractures, by Konsuk and Kueper (2004) in a dolomite limestone fracture, by Nowamooz et al. (2009) in a sandstone fracture and by Zoorabadi et al. (2015) using artificial 2D profiles. These studies considered a single aperture of the fractures, and did not consider the effect that variations in the relative roughness could have on the critical Reynolds number. In contrast, Qian et al. (2015) presented data suggesting smaller values of the critical Reynolds number and, more importantly, reported a reduction in the critical values as the aperture value increases with a constant surface roughness. However, they mentioned that they could not accurately determine the values of the critical Reynolds numbers.

There are also differing results in experiments studying nonlinear flow in field fractures. Quinn et al. (2011) reported critical Reynolds number mostly below $\mathfrak{R}_{c} \sim 1$, and a clear trend showing an increase in the critical Reynolds number as the hydraulic aperture of the fractures increases. However, there is no information available on the roughness of the fractures. Chen et al. (2015) reported much larger critical values of the Reynolds number $\mathfrak{R}_{c} \sim 25-66$, in an extensive investigation of nonlinear flow in field experiments. We note that field experiments pose significant challenges and limited information is available about the geometry of the fractures. In addition, these studies measure the flow in fracture networks and a direct comparison with results obtained in individual fractures is not possible.

Some studies considered the effect of confining stress on the onset of nonlinear flow. Ranjith and Darlington (2007), for example, mentioned that an increase in the confining pressure acting on the fracture would shift the response to an earlier onset of nonlinear effects. Although no information is provided on the change in the aperture and relative roughness of the fracture it is reasonable to assume that an increase in the confining pressure would reduce the average aperture of the fracture and increase the relative effect of roughness. More recent studies, have investigated the effect of confining pressure more systematically. Zhang and Nemcik (2013) studied nonlinear flow in mated and non-mated sandstone fractures. They did not observe nonlinear behavior in the case of mated fractures, possibly due to the small Reynolds numbers studied. 


\begin{tabular}{|c|c|c|c|c|c|c|c|c|c|c|}
\hline Article & Material & $\mathbf{F}$ & $\mathbf{S}$ & $\mathbf{R}$ & $h_{m}[\mu m]$ & $h_{e f f}[\mu m]$ & $\tilde{\sigma}_{h}$ & $\tilde{\sigma}_{z}$ & $\tilde{\xi} / \widetilde{R}_{p}$ & $\mathfrak{R}_{c}$ \\
\hline Zimmerman et al., (2004) & Sandstone & & & $\cdot$ & 148.9 & $117^{i}$ & 0.47 & - & & $\sim 10^{\circ}$ \\
\hline Konzuk \& Kueper (2004) & Dolomitic Limestone & & $\cdot$ & & 417 & 332 & 0.60 & - & & $\sim 5^{6}$ \\
\hline $\begin{array}{l}\text { Ranjith \& Darlington } \\
(2007)\end{array}$ & Granite & & - & & - & $70^{\circ}$ & - & $15.8^{i}$ & & $\sim 5^{i}$ \\
\hline Ji et al., (2008) & Granite & & & $\cdot$ & 760 & - & - & - & & 15 \\
\hline Nowamooz et al., (2009) & Sandstone & & & $\cdot$ & - & 441 & - & - & & $30^{6}$ \\
\hline Radilla et al., (2013) & Granite & & & $\cdot$ & - & 696 & - & - & & $25^{i}$ \\
\hline Qian et al., (2015) & Cement and sand & & $\cdot$ & & $500-2000$ & - & - & - & & $\lesssim 5^{i * 66}$ \\
\hline \multirow{2}{*}{$\begin{array}{l}\text { Quinn, et al., } \\
\text { (2011) }\end{array}$} & \multirow[b]{2}{*}{ Dolostone } & \multirow[b]{2}{*}{ - } & & & - & 25(smallest) & - & - & & 0.09 \\
\hline & & & & & - & $\begin{array}{c}217 \\
\text { (largest) }\end{array}$ & - & - & & 1.59 \\
\hline $\begin{array}{l}\text { Chen, Hu, et al., } \\
\text { (2015) }\end{array}$ & Sanstone and Granite & • & & & - & - & - & - & & $25-66$ \\
\hline \multirow{4}{*}{$\begin{array}{l}\text { Zhang \& Nemcik } \\
\text { (2013) }\end{array}$} & \multirow{4}{*}{ Sandstone } & & $\cdot$ & & - & $265-200^{i, \neq}$ & - & - & $\tilde{\xi} \approx 5.4-7.1^{i}$ & $3.5-4.5$ \\
\hline & & & $\cdot$ & & - & $215-185^{i, \neq}$ & - & - & $\tilde{\xi} \approx 8.6-9.9^{i}$ & $13.1-17.6$ \\
\hline & & & $\cdot$ & & - & $175-165^{i, \neq}$ & - & - & $\tilde{\xi} \approx 14.2-15.0^{i}$ & $19.3-24.8$ \\
\hline & & & $\cdot$ & & - & $210-170^{i, \neq}$ & - & - & $\tilde{\xi} \approx 19.3-23.7^{i}$ & $6.3-8.6$ \\
\hline $\begin{array}{l}\text { Chen, Zhou, et al., } \\
\text { (2015) }\end{array}$ & Granite & & • & & - & $49.7-2.2^{\ddagger}$ & - & $15-1200^{i}$ & $\tilde{\xi} \approx 60-3700^{i}$ & $0.04-11.74$ \\
\hline \multirow{6}{*}{ Zhou et al. (2015) } & \multirow{4}{*}{ Granite } & & $\cdot$ & & - & $35-2.5^{i, \neq}$ & - & $80-1200$ & $\widetilde{R}_{p} \approx 230-3300^{i}$ & $0.075-9.24$ \\
\hline & & & $\cdot$ & & - & $27.5-2^{i, \neq}$ & - & - & - & $0.12-4.47$ \\
\hline & & & $\cdot$ & & - & $30-2.5^{i, \neq}$ & - & - & - & $0.16-5.11$ \\
\hline & & & $\cdot$ & & - & $37.5-2.5^{i, \neq}$ & - & - & - & $0.039-4.51$ \\
\hline & \multirow{2}{*}{ Sandstone } & & $\cdot$ & & - & $35-10^{i, \neq}$ & - & - & - & $0.19-4.09$ \\
\hline & & & $\cdot$ & & - & $47.5-2.5^{i, \neq}$ & - & $40-730^{i}$ & $\widetilde{R}_{p} \approx 15-245^{i}$ & $0.026-2.98$ \\
\hline \multirow{3}{*}{ Rong et al., (2017) } & \multirow{3}{*}{ Granite } & & $\cdot$ & & - & $278-211^{\neq}$ & - & - & - & $8.61-13.35$ \\
\hline & & & $\cdot$ & & - & $238-176^{\ddagger}$ & - & - & - & $5.46-10.11$ \\
\hline & & & $\cdot$ & & - & $218-174^{\ddagger}$ & - & - & - & $4.18-5.98$ \\
\hline Chen et al. (2019) & Sandstone & & - & & - & $42.5-7.5^{i, \neq}$ & - & - & $\tilde{\xi} \approx 75-750^{i}$ & $0.05-3$ \\
\hline \multirow{2}{*}{$\begin{array}{l}\text { Javadi et al., } \\
\text { (2014) }\end{array}$} & \multirow{2}{*}{ Granite } & & $\cdot$ & & $0^{\dagger}$ (smallest) & - & - & - & - & $\begin{array}{c}0.001 \\
\end{array}$ \\
\hline & & & $\cdot$ & & $1750^{\dagger}$ (largest & - & - & - & - & 25 \\
\hline
\end{tabular}




\begin{tabular}{|r|c|c|c|c|c|c|c|c|c|}
\hline & & ) & & & & \\
\hline Rong et al., (2016) & Granite & & $\bullet$ & & & $35-200^{\circ}$ & - & - & - \\
\hline
\end{tabular}

Table 1: Representative summary of previous work on the critical Reynolds numbers in fractured rocks (F: field fractures; S: simulated model

fracture; R: real laboratory fractures). ${ }^{*}$ The values are estimated based on available information. ${ }^{* *}$ The reported value is an estimated upper bound.

${ }^{\dagger}$ The reported aperture is the maximum vertical displacement measured upon shearing the fracture. At small shear displacements small negative vertical displacements are typically observed. ${ }^{\ddagger}$ The aperture range as well as the range of relative roughness values and critical Reynolds numbers corresponds to increasing confining pressures. 
In the case of unmated fractures, however, they obtained a range of critical Reynolds numbers $\mathfrak{R}_{c} \sim 3.5-25$, depending on sample and confining pressure, but no clear trend was observed. In addition, the sample with the largest values of relative roughness exhibited intermediate values of the critical Reynolds number (see Table 1.)

Zhou et al. (2015) investigated different granite and sandstone fracture specimens and found an initial increase in the critical Reynolds numbers with confining pressure, followed by a decrease in the critical Reynolds numbers at higher confining pressures. They speculated that plastic deformation or brittle damage of the of surface asperities could be responsible for the initial increase in critical Reynolds numbers with increased confining pressure due to a reduction in surface roughness. A similar increase in critical Reynolds numbers at increasing but moderate confining pressures was observed by Rong et al., (2017). Also unexpected are the results reported by Chen et. al (2019) obtained in sandstone fractures, showing that the critical Reynolds number decreases with increasing hydraulic aperture. Overall, the effect of increasing the confining pressure on the fracture geometry is complex, which makes it difficult to isolate the effect of relative roughness, or other geometric parameters, on the onset of nonlinear flow.

In real cases, the aperture and the tortuosity of the flow paths are also affected by shear displacements between opposite surfaces of the fracture, and a few studies have considered the effect of such shear displacements on the onset of nonlinear flow. The observed relation between the magnitude of the shear displacement and the critical Reynolds number is complex, probably due to the non-monotonic change in the average aperture with shear displacement (Javadi et al., 2014; Rong et al., 2016). Javadi et al. (2014) reported a large increase of several orders of magnitude in the critical Reynolds numbers as the shear displacement increases, reaching a value $\mathfrak{R}_{c} \sim 15-25$ for large displacements. They also observed an initial decrease in the critical value with small shear displacements in one case. Rong et al. (2016) also reported an initial decrease in the value of the critical Reynolds number for small shear displacement followed by a significant increase, with values ranging from 1.5 to 13 .

In summary, a wide range of values of the critical Reynolds number in fractures have been reported, depending on the geometry of the fracture. Some systematic studies considered two of the main mechanisms affecting the fracture geometry directly, the confining stress acting on the fracture and the presence of a shear displacement between opposite surfaces of the fractures. However, the effects of contacts, deformation and possible damages to the surfaces complicates the analysis of the results. Here, in a departure from most of the previous studies, we performed a series of experiments using open fractures, in which we control the separation between two identical fracture surfaces and systematically investigate the onset on nonlinear flow. Therefore, we eliminate any possible influence of large surface deformations or fracture damage, on the characteristics of the flow. In this way, we focus on the effects of inertia on the flow and its importance depending on the relative magnitude of the surface roughness. As a result, we are able to demonstrate a direct relationship between the relative magnitude of the roughness of the surface fractures and the critical Reynolds number indicating the onset of nonlinear flow. 


\section{Materials and Methods}

\subsection{Fracture model setup and surface roughness}

The model fracture is first generated numerically from a 2D set of uncorrelated, Gaussian distributed, random numbers, representing an $N \times N$ discretization of the height of a fracture surface without overhangs (Brown \& Scholz, 1985). The Fourier transform of this discretized representation of a surface is then modulated by a decaying function of the frequency, that is, a high wave-number filter. The Fourier transform is then inverted to obtain a fracture surface that presents long-range spatial correlations introduced by the filter. Finally, the surface height is scaled with a constant factor that controls the amplitude of the surface height variations, i. e. the magnitude of the surface roughness. A number of different parameters have been used to characterize the roughness of (fracture) surfaces (Barton \& Choubey, 1977; Poon \& Bhushan, 1995; Wang et al., 2016). Two common parameters are the peak asperity height $\xi$, defined as the peak-to-valley distance, that is the difference between the maximum and minimum heights of the surface (similarly the peak-to-mean distance, $R_{p}$, is also used) and the root-mean-square (rms) roughness $\sigma_{z}$, given by the standard deviation of surface heights (Poon \& Bhushan, 1995; Wang et al., 2016). For our surface fracture, we scale the peak asperity height to $\xi \simeq 15 \mathrm{~mm}$, resulting in a rms roughness $\sigma_{z}=2.8 \mathrm{~mm}$. The dimensions of the surface main plane are $100 \mathrm{~mm} \times 100 \mathrm{~mm}$. A contour map of the generated surface is presented in Figure 1a. In our discussions, we shall consider the relative magnitude of the surface roughness, as characterized by $\tilde{\xi}\left(\widetilde{R}_{p}\right)$ and $\tilde{\sigma}_{z}$, corresponding to the peak asperity height (or peak-to-mean distance) and the rms roughness, respectively, and both normalized by the hydraulic aperture of the fracture $h_{\text {eff }}$.

It is important to note that the above statistical parameters may be scale dependent in real fractures that present long-range spatial correlations (Brown \& Scholz, 1985). On the other hand, they provide a means to compare our work with previous studies, as can be seen from the summary presented in Table 1. Alternatively, a fractal characterization can be provided for some fractures, particularly those exhibiting self-affine surfaces (Brown, 1995; Drazer \& Koplik, 2000). Such self-affine fracture surfaces can be generated using a power-law filter (Drazer \& Koplik, 2000). We also used a power-law filter to generate our surfaces but, unfortunately, with an exponent that is larger than those used to generate fractal surfaces. The final matrix of surface heights used to obtain the 3D-printed fracture surface as well as the corresponding power spectral density showing the high-frequency filter are provided in the supplementary information). 

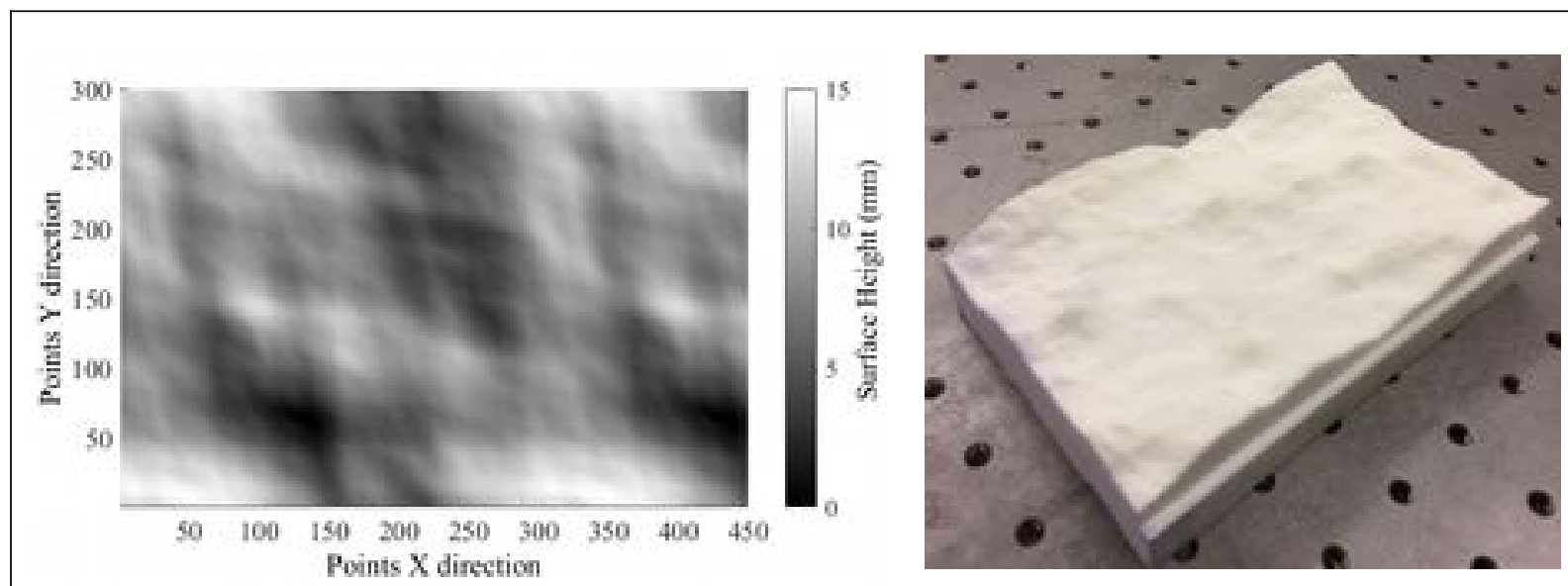

Figure 1: a) Contour map of the surface height. b) 3D printed surface.

The numerically generated surface is then 3D printed with a total length $L_{x}=150 \mathrm{~mm}$, after adding a periodic replica of a portion of the original surface along what would be the flow direction, and a total width $L_{y}=100 \mathrm{~mm}$ (see Figure 1b). A silicon mold of the printed surface is then made, and is used to create a transparent epoxy cast of the surface. A silicon casting is then made from the silicon mold and used to obtain an opaque epoxy cast that mates the transparent epoxy surface. As a result, two mating, epoxy replicas are obtained for the flow experiments. In the experiments the two surfaces are separated by a normal displacement, resulting in a nominally uniform aperture. We note that small deformations could come from the epoxy polymerization process and, as a result, the two surfaces are not expected to perfectly match at small scales (Harold Auradou et al., 2001). In fact, we shall see that contact probably occurs when the vertical separation between the surfaces is of the order of $h_{m} \approx 200 \mu m$, or smaller. On the other hand, the two surfaces do behave as highly correlated, mating surfaces at larger length scales.

\subsection{Flow experiments setup}

A schematic view of the setup used to perform the flow experiments is presented in Figure 2. The opposite surfaces are placed inside an aluminum housing that creates a channel. Surfaces are machined to fit the housing and the final dimensions of the fracture are $L_{x} \times L_{y}=143.5 \mathrm{~mm} \times 79.5 \mathrm{~mm}$. The average aperture is controlled by using flat shims of specified thickness, with the largest value around $h=500 \mu \mathrm{m}$. This is nominally the constant aperture in our fracture but not necessarily the average aperture, due to possible imperfections in the fabrication of the mating surfaces. We note that our model fractures are narrow fractures, where the vertical variations in height are significantly larger than the average aperture in all the experiments considered here. The flow is driven either by a pressure drop imposed using either a Mariotte bottle or a syringe pump, depending on flow rate. We continuously monitor the flow rate, measuring the liquid weight using a collection cup at the outlet of the fracture channel, and the pressure drop, using pressure sensors. We also measure the local pressure inside the fracture with a $5 \times 8$ rectangular array of pressure sensors, with 5 lines of 8 sensors each, parallel to the average flow direction. The parallel lines of sensors are separated a distance $\Delta y=14 \mathrm{~mm}$ in the direction perpendicular to the flow. The sensors in each line are separated a distance $\Delta x=20.5 \mathrm{~mm}$ in the direction of the average flow. Each pressure sensor is connected to a small 
pressure tap drilled through the upper surface of the fracture (see supplementary information for additional drawings).

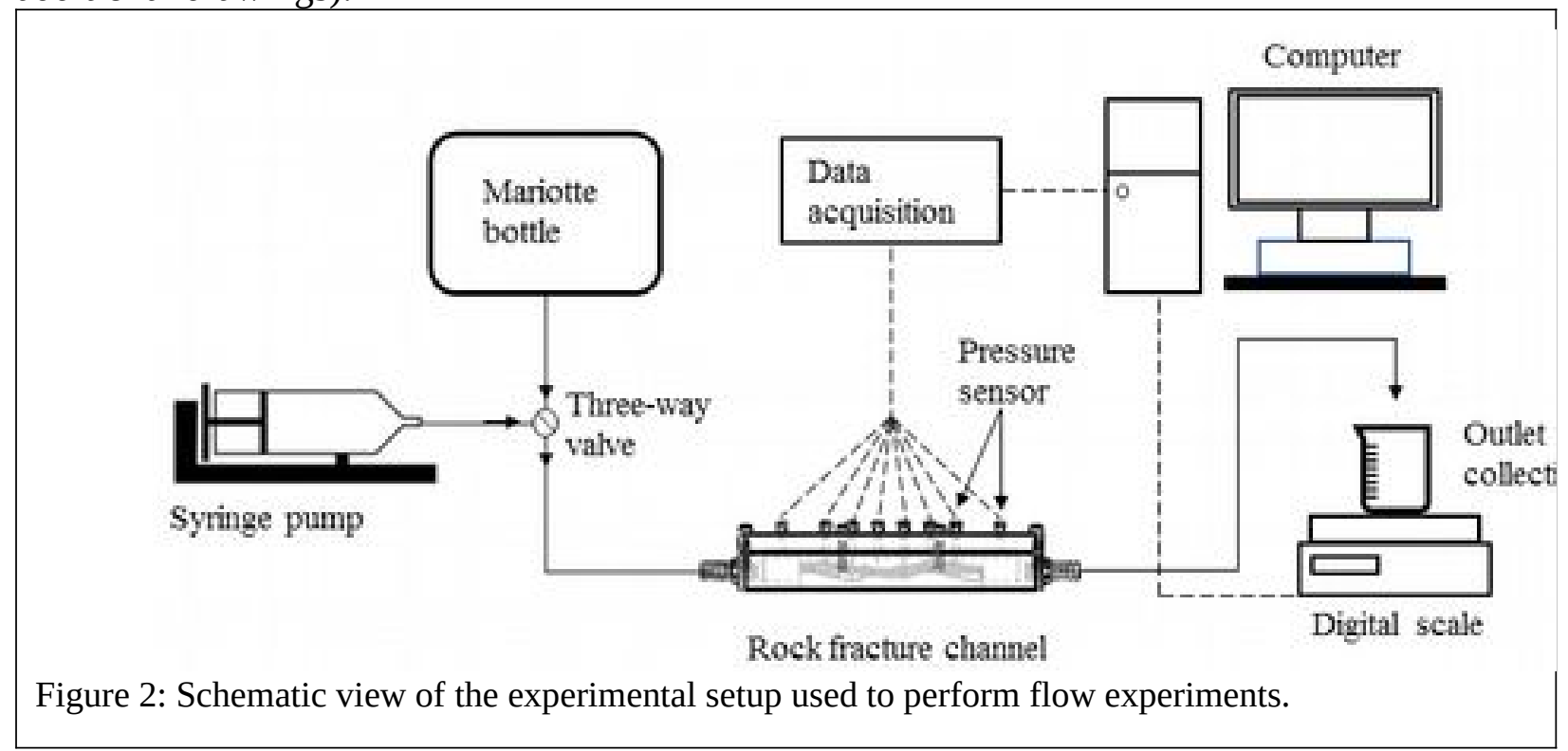




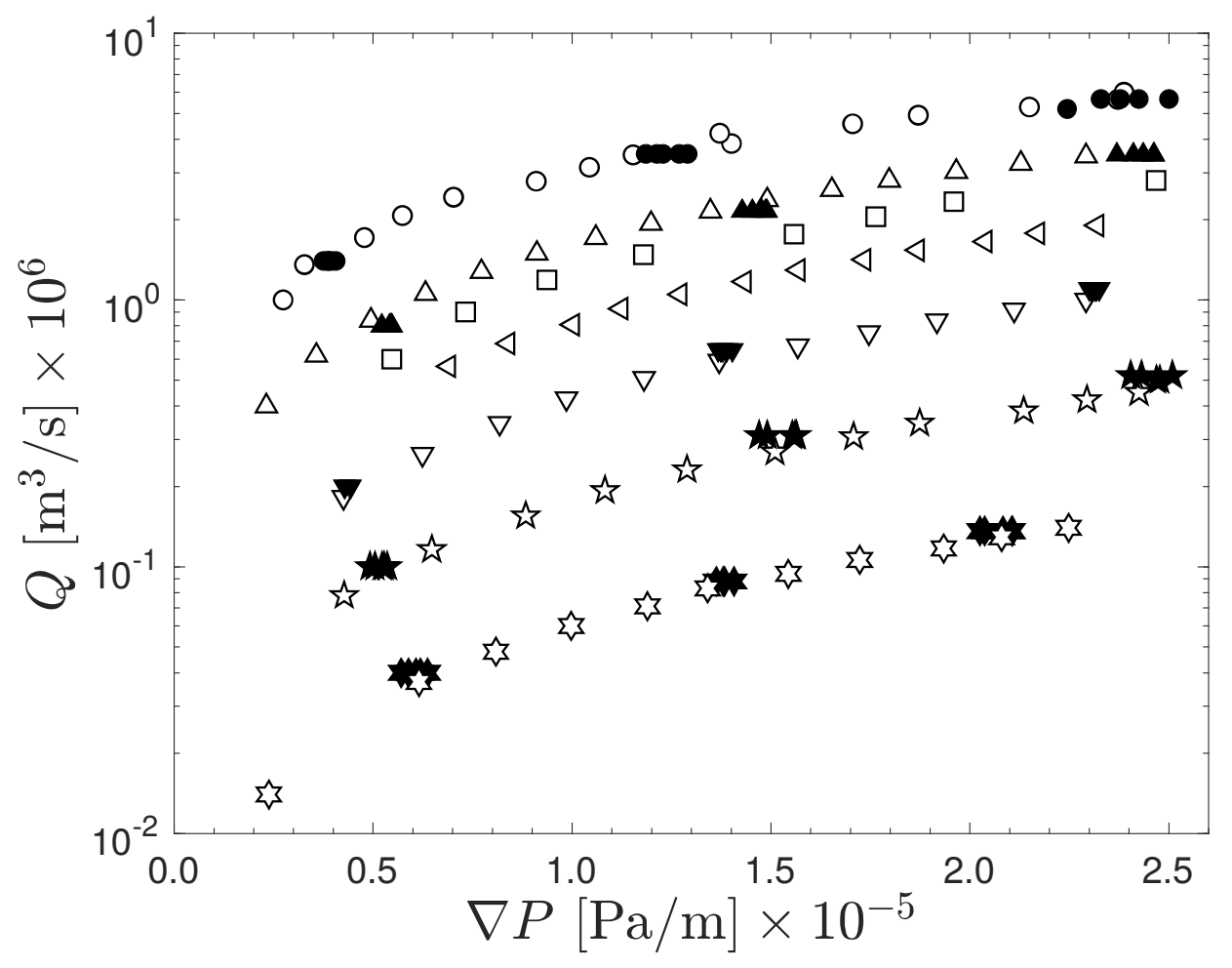

Figure 3: Log-linear plot of the flow rate as a function of pressure gradient for fluids with different viscosity values. The results in this figure correspond to the case when the fracture surfaces are nominally in full contact, that is $h=0 \mu \mathrm{m}$. The measured average aperture is $h_{m}=(270 \pm 30) \mu \mathrm{m}$ and the effective aperture is $h_{\text {eff }}=(190 \pm 10) \mu \mathrm{m}$. The open symbols correspond to measurements from the central line of pressure sensors (obtained with 7 different glycerol aqueous solutions, ranging from pure water (top curve) to $\sim 80 \%$ glycerol by volume (bottom curve)). The solid symbols correspond to experiments measuring the pressure on the entire array of sensors (obtained with 5 different glycerol aqueous solutions). In this case, each group of points corresponds to measurements of the total pressure drop from each of the five lines of sensors parallel to the flow.

\subsection{Properties of the liquids}

In all the experiments, we used mixtures of water and glycerin (99\% Glycerin, McMaster-Carr). The viscosity of these aqueous solutions was controlled by setting specific ratios of glycerin and water (Segur \& Oberstar, 1951; Cheng, 2008). We also measured the viscosity and density of the fluid before each experiment, and those are the values used to report the results. The viscosity of the different solutions ranged from $\mu=0.95 \mathrm{to} 77 \mathrm{mPa} \cdot \mathrm{s}$ and the density of these solutions ranged from $\rho=995 \mathrm{to} 1210 \mathrm{~kg} / \mathrm{m}^{3}$. We performed experiments over a wide range of Reynolds numbers, covering several orders of magnitude $0.001 \leq \mathfrak{R} \leq 500$. For example, in Figure 3 we present the case where no separation is added between the two fracture surfaces, that is, when the fractures are nominally in full contact, $h=0$. For perfectly mating fracture surfaces this would result in a closed fracture and zero flow. However, as discussed above, our surfaces do not perfectly mate and the fracture is effectively open to flow. We present different sets of results corresponding to experiments performed with different glycerol aqueous solutions. We use different solutions in 
order to cover a wide range of Reynolds numbers. The results reported in the figure correspond to approximately 100 independent experiments obtained for a single aperture of the fracture. From these sets of data, we obtain a single value of the effective aperture and critical Reynolds numbers. Similar sets of data are obtained for each of the apertures investigated here.

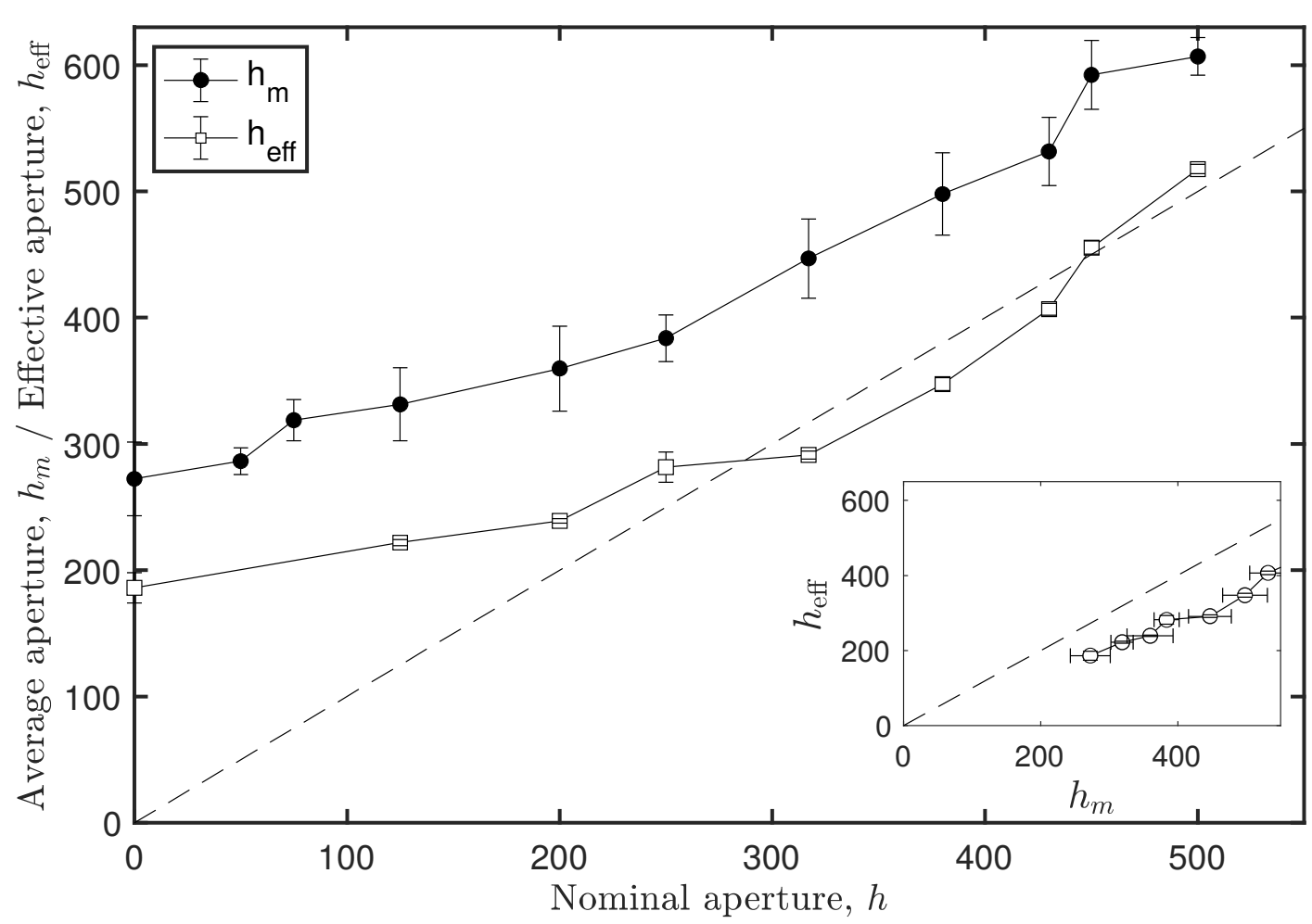

Figure 4: Average aperture, $h_{m}$, and effective aperture, $h_{\text {eff }}$, as a function of the nominal aperture between the two surfaces of the fracture, $h$.

\section{Results and discussion}

\subsection{Average and effective apertures}

As discussed in the introduction, we performed experiments systematically varying the aperture and, as a result, modifying the relative magnitude of the surface roughness. We also mentioned that the nominal separation between the surfaces is not necessarily the same as the average aperture. Therefore, we first determine the average aperture, calculated from the volume inside the fracture, as a function of the nominal aperture. The volume is measured by injecting dyed water and monitoring the advancing front until the entire fracture, placed vertically, is filled with the fluid. The results are presented in Figure 4 . First, it is clear that the average aperture $h_{m}$ is slightly larger than the nominal separation $h$. Specifically, a nearly constant difference of approximately $120 \mu \mathrm{m}$ is observed for nominal separations $h \geq 250 \mu \mathrm{m}$. We can therefore assume that the magnitude of the mating error in the process used to create the fracture model is of the order of $100 \mu \mathrm{m}$. In fact, we also observe a clear change in behavior for nominal separations $h \approx 250 \mu \mathrm{m}$. Specifically, the change in the average aperture becomes 
shallower with a decrease in nominal separation bellow $h \approx 250 \mu m$. This suggests that contact between the two surfaces occurs at $h \approx 250 \mu \mathrm{m}$. In what follows, we use the measured average aperture as a better indication of the fracture aperture compared to the nominal separation between the surfaces.

We then characterize the permeability of the fracture in the linear regime and its corresponding effective aperture. First, the data obtained for a given aperture in multiple experiments using different fluids are made dimensionless as shown in Eq. (5), but using the average aperture $h_{m}$ instead of the nominal aperture value $h$, that is:

$$
\frac{\rho h_{m}^{3}}{12 \mu^{2}} \nabla p=\widetilde{\nabla p}=\left(\frac{h_{m}}{h_{\text {eff }}}\right)^{3}\left(\mathfrak{R}+\beta \mathfrak{R}^{2}\right) \text {. }
$$

The data obtained for each aperture value with different fluids now collapses into a single curve, as expected. This is shown in Figure 5, which corresponds to the same data already presented in dimensional form in Figure 3 above, but now plotted in nondimensional variables. 


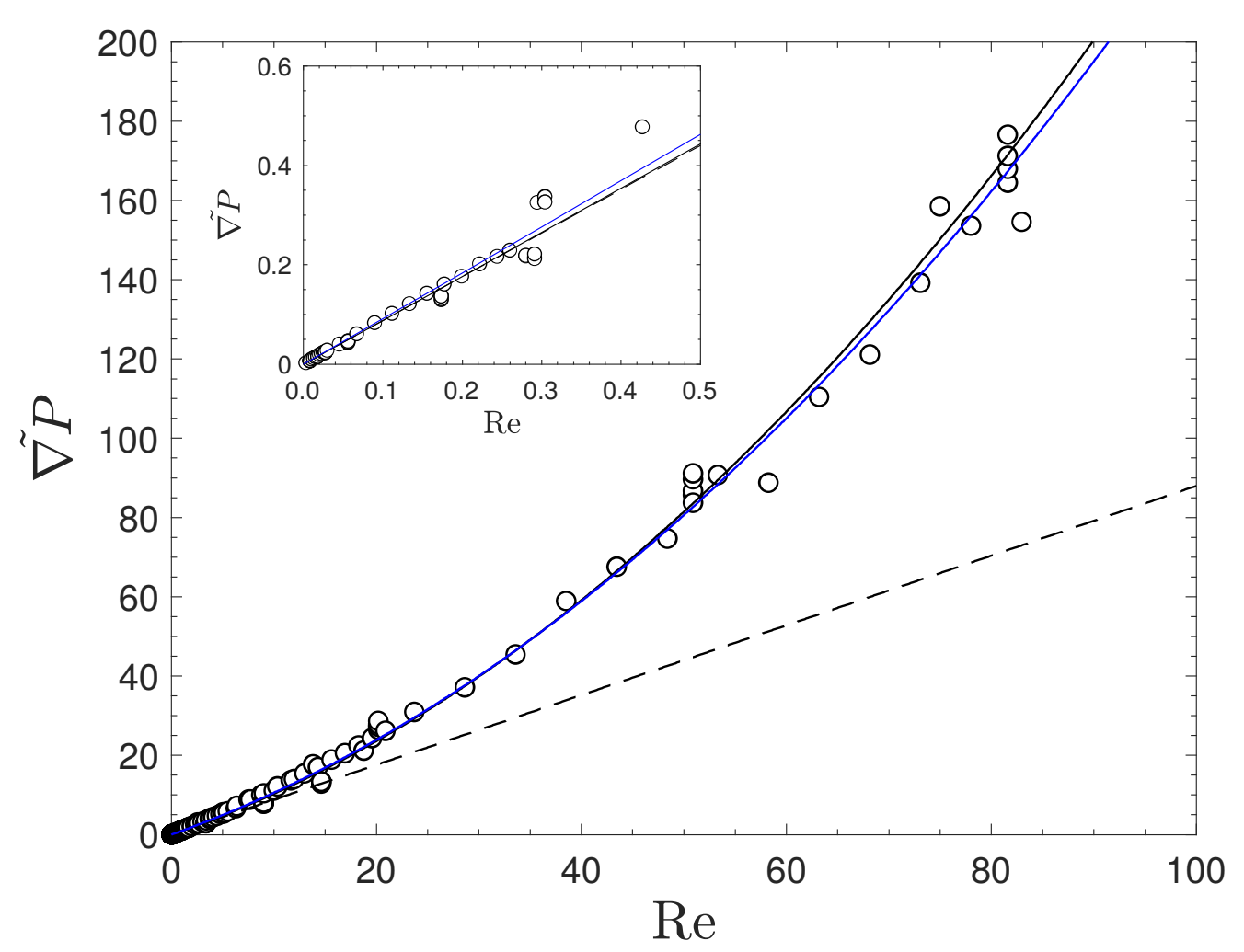

Figure 5: Nondimensional pressure gradient as a function of the Reynolds number for the case of nominally mated surfaces, that ish $=0 \mu \mathrm{m}$. The experimental data is the same as that plotted in Figure 3, made nondimensional as in Eq. (7). The dashed line corresponds to a linear fit (with no intercept) in the region $\mathfrak{R}<0.5$, shown in detail in the inset. The solid lines correspond to quadratic fits. The lighter (blue) line corresponds to a quadratic fit with no intercept. The dark (black) line corresponds to a quadratic fit with no intercept and a fixed linear term obtained from the linear fit for $\mathfrak{R}<0.5$.

We fit a linear dependence of the pressure gradient on the Reynolds number for relatively small values of the Reynolds number, $\mathfrak{R}<0.5$. The data in this range of Reynolds numbers and the corresponding linear fit are shown in the inset in Figure 5. The linear fit determines the effective hydraulic aperture of the fracture and the results are presented in Figure 4. First, we observe that the effective hydraulic aperture is smaller than the average aperture, as usually observed in porous media. Then, it is also clear that the trend of the hydraulic aperture as a function of the nominal aperture closely follows that of the average aperture. That is, the effective hydraulic aperture also shows a nearly constant region for $h \lesssim 250 \mu \mathrm{m}$, consistent with the presence of contacts between the opposite surfaces of the fracture, and a clear linear increase above that value. The relation between the effective and average apertures is shown explicitly in the inset to Figure 4 and shows a nearly linear relation over the range of nominal separations considered in this work.

\subsection{Spatial variations in pressure}

We also looked into the spatial fluctuations of the pressure field at the local level. In Figure 6a we first present the local deviations from a linear profile along the central line of sensors. Specifically, if $p_{i}$ is the pressure at the $i$-th sensor in the central line, we 
normalize the pressure such that $p_{1}=p_{\text {inlet }}=1$ and $p_{8}=p_{\text {outlet }}=0$, and subtract a linear profile between inlet and outlet from the pressure values measured at each sensor. We observe that the relative magnitude of the deviations from a linear profile decreases as the aperture increases.
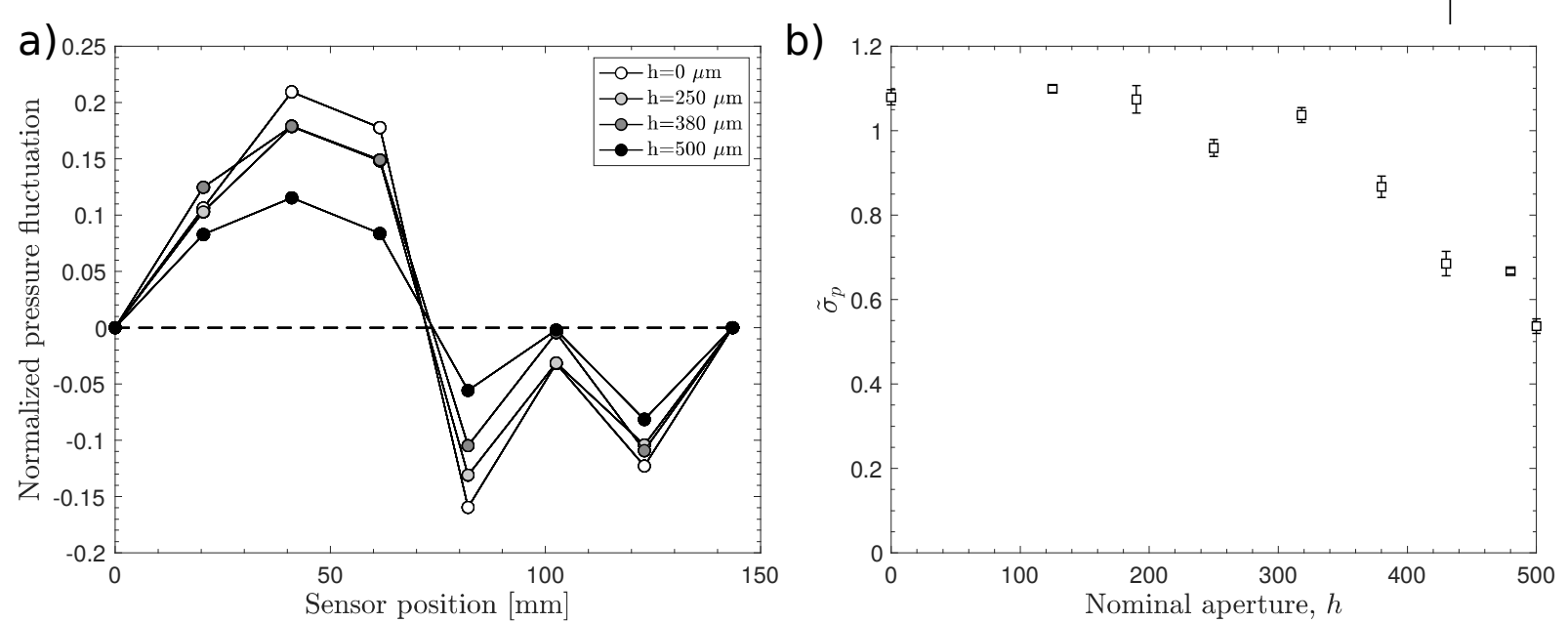

Figure 6: Spatial variation of the pressure field for different apertures. a) Normalized deviation of the pressure field along the flow direction with respect to a linear gradient; $b$ ) Normalized local pressure drop variance as a function of the nominal aperture, $h$.

In order to get a representative magnitude of these spatial fluctuations of the pressure, we calculate the variance in the local pressure gradient, represented by the pressure drop between consecutive sensors in a given line. Specifically, from the $n=8$ pressure sensors in a given line along the flow direction, we first calculate the local pressure drops between sensors, $\Delta p_{i}=p_{i+1}-p_{i}$. Then, we calculate the variance $\sigma_{p}^{2}=\left\langle\Delta p_{i}^{2}\right\rangle_{i}-\left\langle\Delta p_{i}\right\rangle_{i}^{2}$, where $(\cdot\rangle_{i}$ is the spatial average along the line of sensors. In order to average the variance over different experiments we normalized it by the average local pressure drop, $\sigma_{p} / \Delta p$, where $\Delta p=\left\langle\Delta p_{i}\right\rangle$ is the average pressure drop between two consecutive sensors. Note that, in principle, the normalized variance is constant in the linear regime at low Reynolds numbers. Therefore, we calculate the average over all experiments with relatively small values of the Reynolds numbers $(\Re<0.5), \quad \tilde{\sigma}_{p}=\left|\sigma_{p} / \Delta p\right\rangle$, where $\langle\cdot\rangle$ now indicates the average over independent experiments.

In Figure 6b, we present the results on the spatial fluctuations of the local pressure drops as a function of the aperture for the central line of sensors. We observe a trend that is analogous to the variation in the effective aperture, in that the change in pressure drop fluctuations is relatively small for nominal apertures $h \lesssim 250 \mu \mathrm{m}$. This corresponds to the range of nominal apertures in which the effective aperture is relatively constant, possibly due to contacts. In contrast, for larger values of the nominal aperture $(h>250 \mu \mathrm{m})$, there is a significant decrease in the pressure drop fluctuations. 


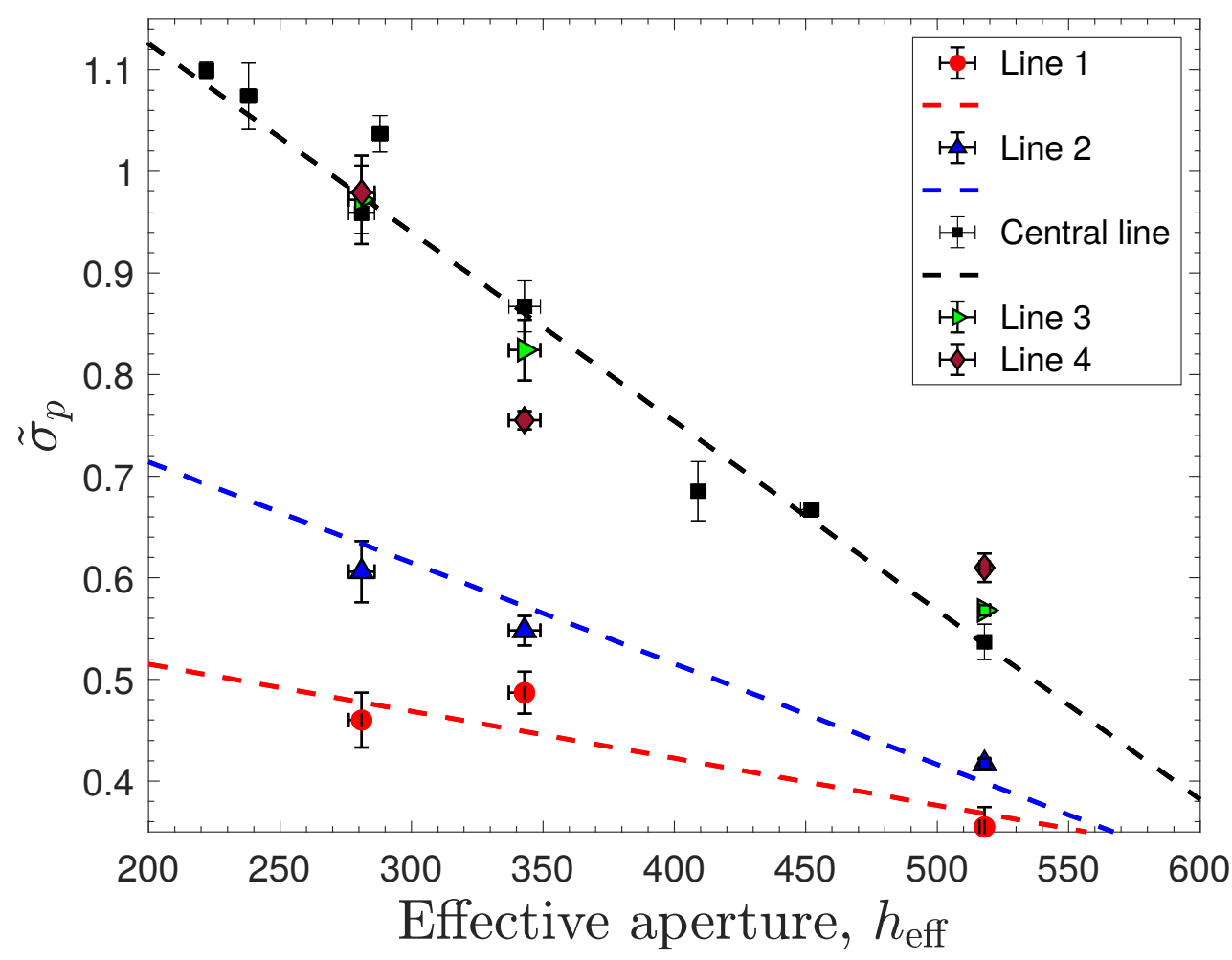

Figure 7: Normalized local pressure drop variance as a function of the effective aperture, $h_{\text {eff }}$. The different symbols correspond to results for different lines of sensors along the flow direction. The dashed lines are linear fits to the data.

This is confirmed in Figure 7, where the normalized spatial fluctuations of the local pressure drop are plotted as a function of the effective aperture. In this case, we observe a clear decrease in the fluctuations over the entire range of values of the effective aperture. We also show a linear decrease that compares well with the measurements. The same trend is observed for all 5 parallel lines of sensors along the flow direction. Although some of these lines of sensors present a smaller magnitude of the relative fluctuations in pressure, they all present a clear reduction in the fluctuations as the effective aperture increases.

These results show that, as the aperture becomes larger, the tortuosity of the flow become smaller and the relative fluctuations in the pressure drop are reduced. Although these measurements provide valuable insight into the flow field, it is important to point out their limitations. First, the length scale over which the pressure drops are measured is arbitrarily fixed by the experimental setup and, second, the measurement is intrinsically one-dimensional.

\subsection{Nonlinear flow and critical Reynolds number}

The flow data in the entire range of Reynolds numbers is fitted with a quadratic equation as given in Eq. (7). First, we only fit the coefficient to the nonlinear term, using the same effective hydraulic aperture as determined by the linear fit, i. e. only fitting the parameter $\beta$ in Eq. (7). Second, we fit the data with a quadratic equation and determine both $h_{\text {eff }}$ and $\beta$ in Eq. 
(7). Both fits are shown in Figure 5 for the data corresponding to nominally mated surfaces. It is clear that both quadratic fits describe the dependence of the pressure gradient on the Reynolds number reasonably well and both give similar results. The corresponding values of the critical Reynolds number for each aperture are presented as a function of the relative rms roughness in Figure 8. A clear trend is observed, with the critical Reynolds number rapidly decreasing as a function of the relative rms roughness. In fact, a decrease proportional to $\tilde{\sigma}_{z}^{-2}$ is presented in Figure 8 and compares well with the reduction in critical Reynolds number observed in the experimental data.

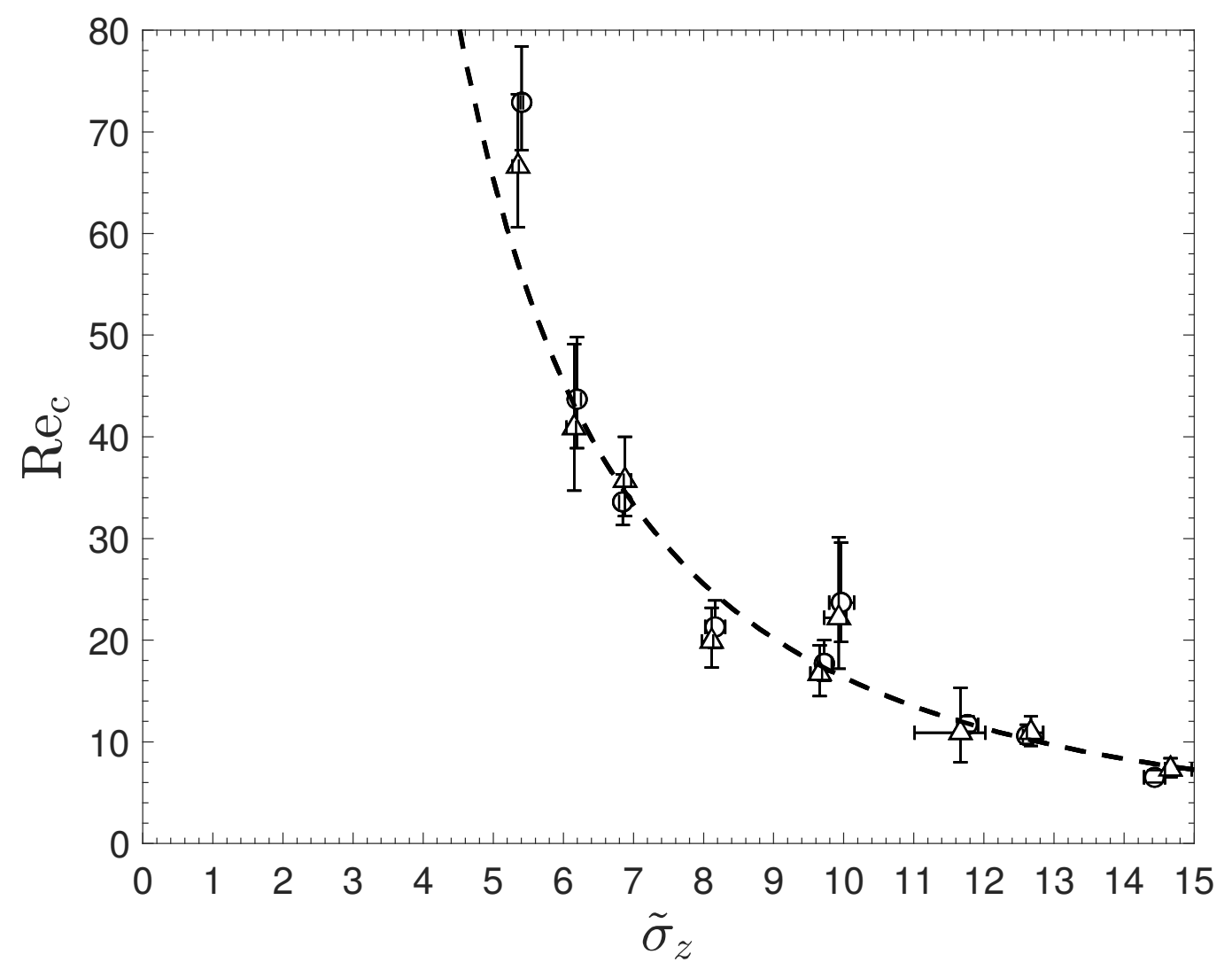

Figure 8: Critical Reynolds number $\left(\mathfrak{R}_{c} \dot{i}\right.$ as a function of the relative surface roughness $\left(\tilde{\sigma}_{z}=\sigma_{z} / h_{\text {eff }}\right)$. Open circles correspond to the fit with Eq. (7) using the $h_{\text {eff }}$ value determined from the linear regime. Open triangles correspond to a quadratic fit with Eq. (7) and both $h_{\text {eff }}$ and $\beta$ taken as free parameters. The dashed line is a fit with to $\mathfrak{R}_{c}=1.6 \times 10^{3} \widetilde{\sigma}_{z}^{-2}$.

These results show that the critical Reynolds number changes significantly depending on the relative magnitude of the surface roughness, decreasing an order of magnitude as the relative surface roughness triples. Our range of relative surface roughness complements the values found in the literature ( $\tilde{\sigma}_{z}>15$ in Table 1$)$ and the results are consistent (e. g. in Table 1 we estimate $\Re_{c}$ 5-10 when the roughness is $\tilde{\sigma}_{z} \approx 15$ ). 


\section{Conclusions}

We have designed and performed a large number of independent flow experiments to characterize both the Stokes regime as well as the onset of inertial effects in an open fracture. Systematically varying the separation between the two mating surfaces of the fracture we investigated the effects that the relative magnitude of surface roughness has on the characteristics of the flow field, including spatial fluctuations of the pressure and the onset of nonlinear flow due to inertia.

First, we performed static measurements of the average aperture while controlling the nominal separation between the two surfaces of the fracture and concluded that small nominal apertures probably result in contact $(h<250 \mu \mathrm{m} \dot{\mathrm{i}}$. This conclusion was supported by the trend observed in the average aperture, which shows a nearly constant value for small separations, with a weak dependence of the average aperture on the nominal separation for $h \lesssim 250 \mu m$. This is consistent with the trend observed in the measurements of effective aperture, that shows a nearly constant effective aperture $h_{\text {eff }} 200 \mu \mathrm{m}$ for small nominal apertures. In fact, the effective aperture shows an almost linear dependence on the average aperture over the range of nominal separations considered in this work. We observe a similar change in behavior in the spatial fluctuations in pressure. We considered the normalized variance in the local pressure drops and observed that it remains nearly constant for small nominal separations $(h<250 \mu \mathrm{m} \dot{\mathrm{a}}$ and decreases for larger separations. Moreover, the normalized variance shows a linear decrease for the entire range of separations when plotted as a function of the effective aperture. Local pressure deviations from a linear profile exhibit a similar behavior, in that they decrease as the relative magnitude of surface roughness decreases. Although expected, this confirms that increased surface roughness results in larger deviations in the local pressure gradients from a uniform and linear profile.

For all nominal apertures, we also explored the nonlinear regime, when pressure gradient grows faster than linear due to inertial effects. In all cases, the Forchheimer equation describes the experimental data reasonably well and provides an empirical value of the critical Reynolds number for each nominal aperture. The advantage of the Forchheimer description is that the critical Reynolds number is a dimensionless number that depends only on the geometry. Our results show that the critical Reynolds number decreases significantly as the relative roughness of the fracture increases. In fact, a factor of 3 increase in roughness results in an order of magnitude decrease in the critical Reynolds number.

Overall, all the results point in the same direction: as the relative roughness decreases, the fracture approaches the geometry of two parallel plates, the pressure gradient approaches a uniform value throughout the fracture and the inertial effects are not present until larger Reynolds numbers are reached. Although our work was performed on a single fracture, an indication of the generality of our results is that similar behavior for the pressure fluctuations was observed along independent lines of pressure sensors. However, more work is clearly needed to investigate the behavior of the pressure fluctuations and critical Reynolds numbers in different fracture surfaces, including experiments that, in contrast to our work, maintain the average aperture while exploring different magnitudes of the surface roughness.

The results presented suggest that studying the onset of inertial effects in fracture flows offers a relatively simple and non-invasive characterization method that can be performed above ground. The critical Reynolds number provides complementary information to that given by the 
effective aperture. In particular, the critical Reynolds number, in combination with the hydraulic aperture could be a sensitive metric for assessing the relative magnitude of the surface roughness in underground fractures and inform transport models used in such systems.

\section{Acknowledgements}

The research reported in this publication is, in part, based upon work done while SSZ is serving at the U.S. National Science Foundation and supported by the NSF Independent Research and Development Program. Any opinion, findings, and conclusions or recommendations expressed in this material are those of the authors and do not necessarily reflect the views of the NSF. Data archiving is underway using the institutional repository at Rutgers university (RUresearch). 


\section{References}

Al-Yaarubi, A. H. B. (2003). Numerical and experimental study of fluid flow in a rough-walled rock fracture (Ph.D dissertation). Imperial College, London. Retrieved from http://spiral.imperial.ac.uk/handle/10044/1/61130

Aminpour, M., Galindo-Torres, S. A., Scheuermann, A., \& Li, L. (2018). Pore-Scale Behavior of Darcy Flow in Static and Dynamic Porous Media. Physical Review Applied, 9(6), 064025. https://doi.org/10.1103/PhysRevApplied.9.064025

Auradou, H., Drazer, G., Hulin, J. P., \& Koplik, J. (2005). Permeability anisotropy induced by the shear displacement of rough fracture walls. Water Resources Research, 41(9), n/a-n/a. https://doi.org/10.1029/2005WR003938

Auradou, H., Drazer, G., Boschan, A., Hulin, J. P., \& Koplik, J. (2006). Flow channeling in a single fracture induced by shear displacement. Geothermics, 35(5-6), 576-588.

Auradou, Harold, Hulin, J.-P., \& Roux, S. (2001). Experimental study of miscible displacement fronts in rough self-affine fractures. Physical Review E, 63(6), 066306. https://doi.org/10.1103/PhysRevE.63.066306

Barton, N., \& Choubey, V. (1977). The shear strength of rock joints in theory and practice. Rock Mechanics Felsmechanik MPanique Des Roches, 10(1-2), 1-54. https://doi.org/10.1007/BF01261801

Bear, J. (1988). Dynamics of fluids in porous media. Courier Dover Publications.

Brown, S. R. (1987). Fluid flow through rock joints: The effect of surface roughness. Journal of Geophysical Research: Solid Earth, 92(B2), 1337-1347. https://doi.org/10.1029/JB092iB02p01337 
Brown, S. R. (1995). Simple mathematical model of a rough fracture. Journal of Geophysical Research: Solid Earth, 100(B4), 5941-5952. https://doi.org/10.1029/94JB03262

Brown, S. R., \& Scholz, C. H. (1985). Broad bandwidth study of the topography of natural rock surfaces. Journal of Geophysical Research: Solid Earth, 90(B14), 12575-12582. https://doi.org/10.1029/JB090iB14p12575

Chen, Y., Lian, H., Liang, W., Yang, J., Nguyen, V. P., \& Bordas, S. P. A. (2019). The influence of fracture geometry variation on non-Darcy flow in fractures under confining stresses. International Journal of Rock Mechanics and Mining Sciences, 113, 59-71. https://doi.org/10.1016/j.ijrmms.2018.11.017

Chen, Y.-F., Hu, S.-H., Hu, R., \& Zhou, C.-B. (2015). Estimating hydraulic conductivity of fractured rocks from high-pressure packer tests with an Izbash’s law-based empirical model. Water Resources Research, 51(4), 2096-2118. https://doi.org/10.1002/2014WR016458

Chen, Y.-F., Zhou, J.-Q., Hu, S.-H., Hu, R., \& Zhou, C.-B. (2015). Evaluation of Forchheimer equation coefficients for non-Darcy flow in deformable rough-walled fractures. Journal of Hydrology, 529, 993-1006. https://doi.org/10.1016/j.jhydrol.2015.09.021

Cheng, N.-S. (2008). Formula for the Viscosity of a Glycerol-Water Mixture. Industrial \& Engineering Chemistry Research, 47(9), 3285-3288. https://doi.org/10.1021/ie071349z

Drazer, G., \& Koplik, J. (2000). Permeability of self-affine rough fractures. Physical Review Letters, 62(6), 8076-8085.

Drazer, G., \& Koplik, J. (2002). Transport in rough self-affine fractures. Physical Review E, 66(2), 026303. 
Gangi, A. F. (1978). Variation of whole and fractured porous rock permeability with confining pressure. International Journal of Rock Mechanics and Mining Sciences \& Geomechanics Abstracts, 15(5), 249-257. https://doi.org/10.1016/0148-9062(78)90957-9

Javadi, M., Sharifzadeh, M., Shahriar, K., \& Mitani, Y. (2014). Critical Reynolds number for nonlinear flow through rough-walled fractures: The role of shear processes. Water Resources Research, 50(2), 1789-1804. https://doi.org/10.1002/2013WR014610

Ji, S.-H., Lee, H.-B., Yeo, I. W., \& Lee, K.-K. (2008). Effect of nonlinear flow on DNAPL migration in a rough-walled fracture. Water Resources Research, 44(11), W11431. https://doi.org/10.1029/2007WR006712

Konzuk, J. S., \& Kueper, B. H. (2004). Evaluation of cubic law based models describing singlephase flow through a rough-walled fracture. Water Resources Research, 40(2). https://doi.org/10.1029/2003WR002356

Macdonald, I. F., El-Sayed, M. S., Mow, K., \& Dullien, F. A. L. (1979). Flow through Porous Media-the Ergun Equation Revisited. Industrial \& Engineering Chemistry Fundamentals, 18(3), 199-208. https://doi.org/10.1021/i160071a001

Mourzenko, V. V., Thovert, J.-F., \& Adler, P. M. (2018). Conductivity and Transmissivity of a Single Fracture. Transport in Porous Media, 123(2), 235-256. https://doi.org/10.1007/s11242-018-1037-y

National Research Council (Ed.). (1996). Rock fractures and fluid flow: contemporary understanding and applications. National Academies Press.

Nowamooz, A., Radilla, G., \& Fourar, M. (2009). Non-Darcian two-phase flow in a transparent replica of a rough-walled rock fracture. Water Resources Research, 45(7), W07406. https://doi.org/10.1029/2008WR007315 
Poon, C. Y., \& Bhushan, B. (1995). Comparison of surface roughness measurements by stylus profiler, AFM and non-contact optical profiler. Wear, 190(1), 76-88. https://doi.org/10.1016/0043-1648(95)06697-7

QIAN, J., WANG, M., ZHANG, Y., YAN, X., \& ZHAO, W. (2015). Experimental study of the transition from non-Darcian to Darcy behavior for flow through a single fracture. Journal of Hydrodynamics, Ser. B, 27(5), 679-688. https://doi.org/10.1016/S10016058(15)60530-3

Quinn, P. M., Cherry, J. A., \& Parker, B. L. (2011). Quantification of non-Darcian flow observed during packer testing in fractured sedimentary rock. Water Resources Research, 47(9). https://doi.org/10.1029/2010WR009681

Radilla, G., Nowamooz, A., \& Fourar, M. (2013). Modeling Non-Darcian Single- and TwoPhase Flow in Transparent Replicas of Rough-Walled Rock Fractures. Transport in Porous Media, 98(2), 401-426. https://doi.org/10.1007/s11242-013-0150-1

Ranjith, P. G., \& Darlington, W. (2007). Nonlinear single-phase flow in real rock joints. Water Resources Research, 43(9). https://doi.org/10.1029/2006WR005457

Rong, G., Yang, J., Cheng, L., \& Zhou, C. (2016). Laboratory investigation of nonlinear flow characteristics in rough fractures during shear process. Journal of Hydrology, 541, Part B, 1385-1394. https://doi.org/10.1016/j.jhydrol.2016.08.043

Rong, G., Hou, D., Yang, J., Cheng, L., \& Zhou, C. (2017). Experimental study of flow characteristics in non-mated rock fractures considering 3D definition of fracture surfaces. Engineering Geology, 220, 152-163. https://doi.org/10.1016/j.enggeo.2017.02.005 
Schrauf, T. W., \& Evans, D. D. (1986). Laboratory Studies of Gas Flow Through a Single Natural Fracture. Water Resources Research, 22(7), 1038-1050. https://doi.org/10.1029/ WR022i007p01038

Segur, J. B., \& Oberstar, H. E. (1951). Viscosity of Glycerol and Its Aqueous Solutions. Industrial \& Engineering Chemistry, 43(9), 2117-2120. https://doi.org/10.1021/ie50501a040

Skjetne, E., Hansen, A., \& Gudmundsson, J. S. (1999). High-velocity flow in a rough fracture. Journal of Fluid Mechanics, 383, 1-28. https://doi.org/10.1017/S0022112098002444

Talon, L., Auradou, H., \& Hansen, A. (2010). Permeability estimates of self-affine fracture faults based on generalization of the bottleneck concept. Water Resources Research, 46(7), W07601. https://doi.org/10.1029/2009WR008404

Talon, Laurent, Auradou, H., \& Hansen, A. (2010). Permeability of self-affine aperture fields. Physical Review E, 82(4), 046108. https://doi.org/10.1103/PhysRevE.82.046108

Wang, M., Chen, Y.-F., Ma, G.-W., Zhou, J.-Q., \& Zhou, C.-B. (2016). Influence of surface roughness on nonlinear flow behaviors in 3D self-affine rough fractures: Lattice Boltzmann simulations. Advances in Water Resources, 96, 373-388. https://doi.org/10.1016/j.advwatres.2016.08.006

Witherspoon, P. A., Wang, J. S. Y., Iwai, K., \& Gale, J. E. (1980). Validity of Cubic Law for fluid flow in a deformable rock fracture. Water Resources Research, 16(6), 1016-1024. https://doi.org/10.1029/WR016i006p01016

Zhang, Z., \& Nemcik, J. (2013). Fluid flow regimes and nonlinear flow characteristics in deformable rock fractures. Journal of Hydrology, 477, 139-151. https://doi.org/10.1016/j.jhydrol.2012.11.024 
Zhou, J.-Q., Hu, S.-H., Fang, S., Chen, Y.-F., \& Zhou, C.-B. (2015). Nonlinear flow behavior at low Reynolds numbers through rough-walled fractures subjected to normal compressive loading. International Journal of Rock Mechanics and Mining Sciences, 80, 202-218. https://doi.org/10.1016/j.ijrmms.2015.09.027

Zimmerman, R. W., Al-Yaarubi, A., Pain, C. C., \& Grattoni, C. A. (2004). Non-linear regimes of fluid flow in rock fractures. International Journal of Rock Mechanics and Mining Sciences, 41, 163-169.

Zoorabadi, M., Saydam, S., Timms, W., \& Hebblewhite, B. (2015). Non-linear flow behaviour of rough fractures having standard JRC profiles. International Journal of Rock Mechanics and Mining Sciences, 76, 192-199. https://doi.org/10.1016/j.ijrmms.2015.03.004 